\title{
Avaliação do Nível de Serviço ao Cliente no Contexto do Marketplace da Empresa Alfa
}

\author{
Customer Service Level Assessment in the Context of the Alpha Company Marketplace
}

Evaluación del Nivel de Servicio al Cliente en el Contexto del Marketplace de la Empresa Alfa

\author{
Renata de Souza Franco ${ }^{1}$ \\ Leonardo Caixeta de Castro Maia² \\ Rafael Gontijo de Souza Filho ${ }^{3}$
}

\begin{abstract}
Resumo
Nesse século XXI, impulsionados pela disseminação do acesso à internet, ocorreram avanços tecnológicos exponenciais. Nesse contexto, as operações comerciais e, simultaneamente, o comércio eletrônico contribuíram de forma decisiva para mudanças organizacionais. Assim, o objetivo deste estudo é compreender o processo de serviço ao cliente a partir de uma estratégia de marketplace. Para isso, fez-se um estudo de caso, com questionário pré-estruturado, com uma empresa de comércio eletrônico, destacando-se as decisões ao longo do processo transacional. Diante dos apontamentos, comprovou-se que a estratégia de marketplace está alinhada à definição de que a qualidade no comércio eletrônico é um constructo multidimensional. O artigo tem como limitação o estudo de caso único, que contemplou exclusivamente a perspectiva da empresa focal da cadeia de suprimentos. Os estudos futuros poderão abordar esse tema ampliando a amostra de empresas para a análise - tanto a montante quanto a jusante da empresa focal - e estendendo a pesquisa aos grandes players do marketplace. Destaca-se, também, a contribuição social, tendo em vista as possibilidades para pequenas organizações acessarem mercados com barreiras por questões financeiras, logísticas ou de marketing.
\end{abstract}

Palavras-chave: Estratégia de Cadeia de Suprimentos; Marketplace; Serviço ao Cliente; Estudo de Caso.

\begin{abstract}
In this 21st century, driven by the spread of internet access, exponential technological advances have taken place. In this context, commercial operations and, at the same time, electronic commerce contributed decisively to organizational changes. Thus, the objective of this study is to understand the customer service process based on a marketplace strategy. For this, a case study was carried out, with a pre-structured questionnaire, with an e-commerce company, highlighting the decisions throughout the transactional process. Given the notes, it was proved that the marketplace strategy is aligned with the definition that quality in electronic commerce is a multidimensional construct. The article's limitation is the unique case study, which exclusively contemplated the supply chain focal enterprise perspective. Future studies will be able to address this theme by expanding the sample of companies for analysis - both upstream and downstream of the focal company - and extending the research to major marketplace players. The social contribution also stands out, considering the possibilities for small organizations to access markets with barriers due to financial, logistical, or marketing issues.
\end{abstract}

Keywords: Supply Chain Strategy; Marketplace; Customer service; Case study.

\section{Resumen}

En este siglo XXI, impulsado por la divulgación del acceso a internet, ocurrieron avances tecnológicos exponenciales. En este contexto, las operaciones comerciales y, simultáneamente, el comercio electrónico contribuyeron de forma decisiva para los cambios

19 Mestre em Administração e Bacharel em Ciências Econômicas pela Universidade Federal de Uberlândia. Pós Graduada em Gestão em Marketing pela ESAMC. 
organizacionales. Asi, el objetivo de este trabajo es comprender el proceso de servicio al cliente a partir de una estrategia de Marketplace. Para eso, se hizo un estudio de caso, con cuestionario semiestructurado, con una empresa de comercio electrónico, dando enfoque a las decisiones a lo largo del proceso transaccional. Ante los apuntes, se comprobó que la estrategia de Marketplace está alineada a la definición de que la calidad en el comercio electrónico es un constructo multidimensional. El artículo tiene como limitación el estudio de caso unico, que contempló exclusivamente la perspectiva de la empresa focal - y extendiendo la para pequeñas organizaciones tener acceso a mercados con barreras por cuestiones financieras, logisticas o de marketing.

Palabras clave: Estrategia de Cadena de Suministros; Marketplace; Servicio al Cliente; Estudio de Caso.

\section{Introdução}

O dinamismo do mercado tem aumentado a competição e a incerteza nos setores econômicos, com tendência de aumento na variedade e customização de produtos e, consequentemente, o aumento da incerteza da demanda. Esses fatores proporcionam novos desafios de gestão e novas práticas na forma como as cadeias de suprimentos são projetadas e gerenciadas (Simão \& Gonçalves, 2015)

No Brasil, essa realidade não é diferente. Cada vez mais as empresas dependerão da capacidade gerencial para aumentar sua competitividade, reduzindo custos e aumentando a qualidade em relação aos concorrentes (Guissoni \& Farinha, 2019). Dessa forma, torna-se necessário que as empresas desenvolvam novos modelos de gestão, buscando atender, e até mesmo superar, as expectativas do cliente por meio do aumento na qualidade e produtividade (gerenciamento efetivo dos insumos necessários à produção) e de um modelo efetivo de acompanhamento do desempenho da produção (Durski, 2003).

Buscando melhorar o seu desempenho, empresas de diversos setores têm desenvolvido novas estratégias ggísticas. A excelência nessas atribuições pode melhorar significativamente $\mathrm{o}$ atendimento aos clientes e a eficiência a confiabilidade do processo logístico, ajudando a reduzir custos (Leuschner, Charvet, \& Rogers, 2013).

Ressalta-se que a comunicação, especialmente em relação à informação, e a obtenção de dados e conhecimentos vêm evoluindo constantemente, modificando o comportamento e os hábitos pessoais (Figueira \& Buri, 2017). As novas tecnologias de comunicação proporcionaram facilidades que mudaram os relacionamentos no mundo dos negócios, sobretudo entre empresas e consumidores. Nesse sentido, pode-se destacar como um dos aspectos principais o aumento do volume e complexidade dos processos logísticos, envolvendo a movimentação dos produtos (Yu, Wang, Zhong, \& Huang, 2016).

Nesse contexto, uma das estratégias que vem sendo adotadas é a entrada em um mercado virtual. No Brasil, em 2017, o faturamento do e-commerce totalizou aproximadamente 60 bilhões de reais (Ebit, 2018).

Assim como o físico, esse mercado possui grandes empresas, geralmente líderes em seu segmento, com grande parcela das vendas, que trazem consigo pequenas e médias empresas para vendas via web. Chamado de marketplace, esse é um mercado em que compradores e vendedores encontram-se virtualmente para realizar negócios entre si, e que possui três principais funções: tornar possível o encontro de compradores e vendedores; facilitar a troca de informações, bens, serviços e pagamentos relacionados à transação; e proporcionar uma infraestrutura que possibilite o funcionamento eficiente desse mercado (Cheah, Phau, \& Liang, 2015; Turban, Lee, King, Liang, \& Turban, 2009). Partindo desse ponto de vista, descreve-se o problema de pesquisa: como se dá o processo de serviço ao cliente a partir de uma estratégia de marketplace? Dessa forma, respondendo à pergunta central, o objetivo deste estudo é compreender o processo de serviço ao cliente a partir de uma estratégia de marketplace. Nesse contexto, é necessário desenvolver uma breve revisão sobre as estratégias voltadas para a cadeia de suprimentos e, a partir daí, identificar quais são as práticas realizadas pela organização que permitem colaboração com os fornecedores para viabilizar o marketplace.

Nesse sentido, o artigo está dividido em seis seções, a seguir: após esta introdução, apresenta-se o referencial bibliográfico. No terceiro tópico, avalia-se o método de pesquisa e, no quarto, a ênfase ao estudo de caso, respondendo às perguntas "como" e "porque". O quinto tópico avalia os resultados. Por fim, no sexto tópico, têm-se as considerações finais do artigo.

\section{Referencial Teórico}

Será exposta uma revisão bibliográfica acerca dos seguintes tópicos: uma visão macro acerca das estratégias voltadas para cadeia de suprimentos, fazendo um recorte sobre o comércio eletrônico. Nesse interim, aborda-se a viabilização do marketplace para os agentes da cadeia de suprimentos a montante da empresa considerada focal. Por fim, abordam-se os mecanismos de controle e qualidade do serviço ao cliente.

\subsection{As estratégias na cadeia de suprimentos}

Lambert (2006) afirma que a gestão da cadeia de suprimentos é mais sensivel às mudanças na demanda do cliente final. Fisher (1997) complementa que, como os clientes têm necessidades diferentes e os produtos apresentam demandas específicas, são necessárias diferentes estratégias de cadeia de suprimentos. Nesse contexto, o processo

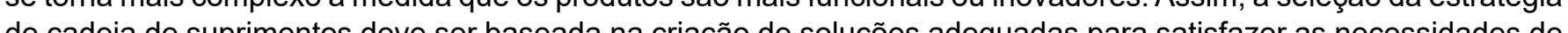
diferen cosmenter

Nesse sentido, este artigo irá abordar três mecanismos que as organizações utilizam como estratégia para garantir a competitividade em cenários de incerteza da cadeia de suprimentos: a estratégia de postergação, os

Van Hoek (2001) afirma que o principal objetivo da estratégia de postergação é atrasar as atividades da cadeia de suprimentos até o último momento possível, mesmo que seja depois do recebimento dos pedidos. Essa postergação pode acontecer na produção e também na logística.

No que se refere à adoção de práticas colaborativas, Gomes e Kliemann (2015) afirmam que as empresas vêm buscando movimentos no sentido de buscar aumentar a competitividade com iniciativas que envolvem arranjos interempresariais, ou seja, em forma de cadeias. Como característica comum aos métodos encontrados está o compartilhamento de informações como fator-chave para melhoria do desempenho.

Whipple e Russel (2007) corroboram essa ideia, afirmando que o desenvolvimento de iniciativas de colaboração na cadeia de suprimentos permite relacionamentos interorganizacionais mais fortes, com a intensificação da troca de informações, o que melhora a qualidade da tomada de decisão, reduz a incerteza da demanda e, em última instância, melhora o desempenho da cadeia de suprimentos.

Além das práticas colaborativas, as cadeias de suprimentos também são influenciadas pelo uso de tecnologias cada vez mais modernas. A evolução tecnológica resulta em diminuição no custo dos equipamentos 2013). Entretanto o simples emprego das tecnologias não garante um fator de diferenciação estratégica, pois elas

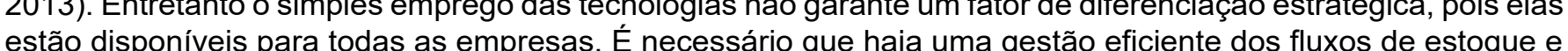
inovação nas operações logísticas (Branski \& Laurindo, 2013).

Dessa forma, é importante garantir que haja uma gestão integrada da logística, de forma que os agentes da cadeia sejam coordenados, permitindo que diversas empresas possam operar como uma única organizaçãa,
favorecendo as práticas colaborativas. Diante desse cenário, um dos duelos consiste em identificar ferramentas que ofereçam melhor viabilidade logística para os negócios (Carvalho \& Campos, 2013).

Assim, para aumentar a competitividade, reduzindo seus custos e melhorando os serviços, é essencial que os agentes sejam integrados, não só em relação a suas diversas atividades logísticas, como transporte, estoque e distribuição, mas também em seus processos com outros agentes da rede, como fornecedores, clientes e transportadoras. A integração decorre da partilha de informação apoiada pelas tecnologias, que colocam as bases para 0 estabelecimento de redes. Portanto, a tecnologia é central para a promoção de colaboração entre as empresas e para estimular a formação de redes (Branski \& Laurindo, 2013). A evolução da tecnologia traz consigo alterações no aumento na concorrência entre as empresas (Pigatto \& Alcantara, 2007).

É importante, também, destacar a importância da existência de alinhamento entre a estratégia e os objetivos da adoção de uma inovação. A estrutura organizacional deve ser adequada à implementação da estratégia, de maneira que

\subsection{Serviço ao cliente e a qualidade do serviço}

O produto entregue aos clientes pode ser um produto acabado ou semiacabado, sendo transferido para outra empresa, que cria valor agregado. Assim, pode haver um único processo envolvido ou vários processos que, juntos, geram a cadeia de suprimentos. Portanto, a cadeia de suprimentos é uma mistura de processos que conecta 2004; Mentzer, Flint, \& Hult, 2001).

tipo de serviço oferecido ao cliente pode apresentar fatores individuais (Davis \& Mentzer, 2006). Nesse contexto, muitos estão sob controle logístico e são classificados quanto aos elementos de pré-transaçãa, transação e pós-transação (Stank, Pellathy, In, Mollenkopf, \& Bell, 2017; Ballou, 1993). Os elementos de pré-transação estão relacionados às políticas ou programas da empresa, que envolvem regras ou estatutos da administração de serviços, adequação da estrutura organizacional e flexibilidade do sistema. Essa parceria deixa claro o serviço oferecido e, principalmente, os planos de contingência. Quanto aos elementos da transação, estão relacionados aos resultados das entregas ao cliente e às variáveis do serviço ao cliente, diretamente envolvidas no desempenho da distribuição 
física, tais como a confiabilidade do produto e da entrega. Por fim, os elementos pós-transação estão relacionados ao apoio do produto no campo, servindo como proteção ao cliente (Ballou, 1993).

A soma de todos esses elementos constitui o nível de serviço. Portanto, medir o desempenho logístico oferecido ao cliente constitui o elemento-chave no desenvolvimento de estratégias logísticas por mensurar o ciclo de pedido desde o momento que é solicitado até a entrega em perfeitas condições aos clientes. Nesse panorama, os gestores podem gerar estatísticas sobre a velocidade, acuracidade e confiabilidade das ordens (Ballou, 1993; Daugherty,

Partindo desses conceitos, podem-se destacar como principais objetivos do desenvolvimento e da implementação de sistemas de avaliação de desempenho: monitorar o nível de serviço e os custos; controlar o processo; redirecionálo, caso necessário, e evitar a ruptura (Aguiar \& Sampaio, 2014). Portanto, garantir a confiabilidade do serviço ao qué é consich que e possivel por melo do n \& Vickery, 1999)

Do ponto de vista dos varejistas do comércio eletrônico, a qualidade do serviço consiste em três dimensões: a qualidade do processo, a qualidade do resultado e a recuperação. Isso leva em consideração o design, a precisão da informaçăo, a privacidade, a funcionalidade

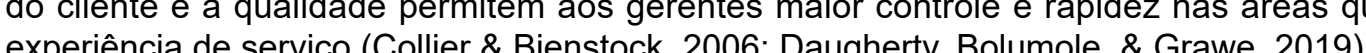
De um lado, os consumidores estão procupad

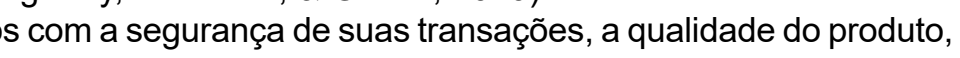
a incerteza em termos de entrega e a perda da função social ou de entretenimento (Hsu, Huang, \& Swanson, 2010; O'Cass \& Carlson, 2012). Por outro lado, os varejistas também enfrentam seus desafios logísticos, de entrega ou de riscos da cadeia de suprimentos (Reinartz, Dallaert, Krafft, Kumar, \& Varandarajan, 2011).

\subsection{0 e-commerce como mecanismo de alavancagem de vendas e os marketplaces}

O e-commerce amplia os canais de vendas das empresas, que passaram a usar a internet para distribuir, vender, negociar e prestar serviços. De acordo com Kubota e Milani (2011), o uso da internet como canal de comercialização está associado a elevações do nível de eficiência das empresas.

No caso das pequenas empresas, Mazzali e Padilha (2006) destacam as dificuldades para implementar essa estratégia, principalmente no que se refere à logística, o que poderia ser amenizado por meio de parcerias com empresas maiores.
com

Marques (2010) constatou, através de pesquisas de campo, que a logística está presente nas mais diversas formas de transações eletrônicas, e que a interação entre logística e os diversos setores da empresa é fundamental para o comércio eletrônico, pois, em sua essência, as transações geram vários fluxos de produtos e informações que, uma perfeita integração dos fluxos de informaçõ̂es e produtos ao longo da cadeia, pois é ela em si que viabilizará o nível de serviço desejado a um custo viável.

Com o aumento do número de lojas, é comum que o consumidor se torne mais exigente, pois o espaço para realização de suas compras não se resume apenas a um site com layout bom, envolve vários atributos relacionados, como marketing e logística, os pontos a serem analisados neste artigo. Assim, a qualidade percebida é um componente do valor da marca (Daugherty, Bolumole, \& Grawe, 2019).

Fica evidente, pelos estudos sobre o comércio eletrônico, que os consumidores estabelecem mecanismos para reduzir os riscos financeiros, os riscos psicológicos, o risco de perda de tempo e também o risco logístico atrelado às atividades de entrega ou devolução do produto (Bezes, 2016; Cheah, Phau, \& Liang, 2015).

Cheah et al. (2015) declaram que os estudos sobre comércio eletrônico têm se dividido em três campos: primeiramente, a avaliação do canal de vendas, também denominado shopping channel. O segundo campo de estudos faz referência aos fornecedores bem como as características dos produtos. Por fim, a terceira linha de pesquisa foca nas características do consumidor. Nesse manuscrito, será avaliada a percepção sobre o canal de vendas marketplace e os critérios de qualidade do serviço, as vantagens relativas, a percepção do risco e a segurança, tanto dos dados como do serviço a ser prestado.

Com base na teoria sobre estratégias utilizadas na cadeia de suprimentos e o serviço ao cliente descrita até aqui, observa-se que o modelo de distribuição por marketplace atende aos três mecanismos descritos nesta seção - a estratégia de postergação, os mecanismos de compartilhamento e a tecnologia da informação - para garantir o nível de serviço ao cliente.

modelo de marketplace consiste em um intermediário que, no papel de fornecedor de infraestrutura de mercado, inclui uma comunidade de vendedores fazendo negócios dentro dessa estrutura, que pode ser fisica ou virtual (Turban et al., 2009). Ao permitir que operadores independentes comercializem seus produtos em sua plataforma, a estratégia permite aos grandes players do mercado a ampliação dos sortimentos sem custos de estoque. Essa estratégia já é utilizada por quase metade dos 50 maiores e-commerces do Brasil. Nascida como uma estratégla de amplação de sortimento para empresas multicategorias, ela também vem sendo utilizada por empresas especializadas (SBVC, 2018).
Além da redução dos custos de estoque, o marketplace trouxe várias vantagens para os grandes players, como diversificação de produtos, aumento de receita com melhoria de margens de lucro, além do crescimento de vendas em mercados de nicho. Por outro lado, a estratégia traz também vários desafios operacionais, como a perda de qualidade no serviço de entrega e pos-venda e o alto custo de gestão. No caso dos operadores independentes, 0 modelo permite um rápido aumento de recelta com baixo investimento em markeling, uma vez que utiliza o tráfego e a credibilidade de umá rede varejsta já consolidada no mercado brasileiro, além de reduzir gastos com investimentos em

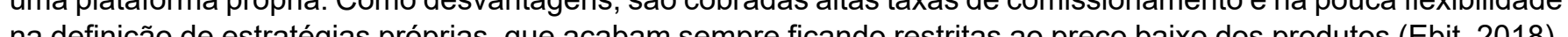
É consumidores finais, facilitando os marketplaces tambem permiram a entrada de fabricantes na venda direta para forma, os fabricantes podem receber as vendas de forma ágil e negociar os seus prazos com os marketplaces diminuindo a neces diminuindo a necessidade de capital de giro e os riscos

A pois pode afetar diretamente o resultado financeiro da empresa, bem como o grau de satisfação e fidelização dos de um produto, são decisivas para a opç̃o de compra em uma loja virtual Diante desse cenário, o o exodelo de (a) marketplace aca terceirizando os processos de entrega de produstos. As

Tanto em pesquisas nacionais como internacionais, destaca-se que o constructo preço já não era considerado como fator ganhador de pedido, pois os produtos anunciados na internet possuem preços semelhantes. Portanto, o consumidor busca alternativas de serviços logísticos e também comerciais para ter satisfação (Bhatnagar \& Ghose, 2004; Collier \& Bienstock, 2006; Vieira, Matos, \& Slongo, 2009).

Embora a contribuição teórica sobre o tema ainda esteja em evolução (Saghiri, Wilding, Mena, \& Bourlakis, 2017), esse novo modelo de negócio vem ganhando espaço no mercado nacional, o que pode ser observado pelos números do setor: considerando as vendas de produtos oferecidos por lojas parceiras, o setor movimentou aproximadamente $\mathrm{R} \$ 8,8$ bilhões no ano de 2017 , o que representa $18,5 \%$ do total de vendas no comércio eletrônico no Brasil (Ebit, 2018).

Entre os motivos para essa evolução, destacam-se: a fácil e rápida integração de lojistas; gestão de qualidade de atendimento e excelência nos processos de gestão de estoque; e o frete e a entrega, que são considerados
fatores fundamentais para o sucesso do modelo de marketplace no país (Ebit, 2018: Vieira, Matos, \& Slongo, 2009).

Na próxima seção será apresentada a metodologia de pesquisa utilizada no desenvolvimento deste trabalho.

\section{Metodologia de Pesquisa}

Este trabalho pode ser considerado uma pesquisa aplicada qualitativa, pois gera conhecimentos para a aplicação prática, visando à solução de problemas específicos, e leva em consideração a interpretação de fenômenos e
atribuições de significados. Do ponto de vista dos seus objetivos, este trabalho classifica-se como exploratório, pois, além de ter por finalidade promover maior profundidade de conhecimento sobre o tema, baseou-se na análise do exemplo da empresa focal na cadeia de suprimentos e suas relações com o mercado para estimular a compreensão do problema de pesquisa (Silva \& Menezes, 2005).

Assim, em primeiro lugar, foi feito um levantamento bibliográfico, em que foram consultadas pesquisas relacionadas ao tema, por meio de artigos, livros e dissertações. A estratégia de pesquisa foi definida com base nas condições presentes no modelo de Yin (2015): i) no tipo de questão de pesquisa proposta; ii) na extensão do controle sobre os eventos comportamentais efetivos do pesquisador e iii) no grau de enfoque. Dessa forma, foi realizado um estudo de caso, que tem como objetivo investigar um fenômeno contemporâneo e reter uma perspectiva holística entrevistas, cuja finalidade é captar explicações e interpretações dessa realidade (Gil, 2002).

Em relação à escolha da empresa Alfa, podem-se destacar dois aspectos que motivaram a sua escolha. Em primeiro lugar, a possibilidade de acesso às informações, em função da localização física. Em seguida, a importância
que representa no mercado de e-commerce no Brasil, por fazer parte de uma empresa atacadista e com relevância no contexto brasileiro (Abad, 2019).

Para possibilitar este estudo, foi realizada uma entrevista semiestruturada com o gestor do marketplace da empresa. A entrevista combinou perguntas sobre as estratégias de nivel de serviço e marketing. O objetivo do protocolo de pesquisa foi delimitar o volume das informaçoes, obtendo-se um direcionamento maior para o tema, intervindo a fim de que os objetivos sejam alcançados (Boni, 2005).

A entrevista foi gravada e, posteriormente, transcrita para um documento de edição de texto. As expressões foram organizadas em uma planilha de dados e categorizadas conforme o assunto discutido em cada uma delas. No que se refere à análise dos dados coletados, foi utilizada a técnica de análise de conteúdo, que, segundo Bardin

Revista Ciências Adiministrativas, 26(1): 9382, 2020 
(1977), tem como objetivo principal o desvendar crítico. Seguindo as principais fases da análise proposta, os dados foram organizados, codificados e categorizados. Por fim, foi realizada a inferência e interpretação das informações. Na próxima seção será apresentado o estudo de caso realizado.

\section{Estudo de Caso}

Com o propósito de compreender e identificar as práticas realizadas para viabilizar o marketplace, foi realizado um estudo de caso com a empresa Alfa, um dos primeiros e-commerces do país, criada por um grupo atacadista, um estudo de caso com a empresa Alfa, um dos primeiros e-commerc
em 2000, para oferecer produtos ao consumidor final (Abad, 2019).

Após sua fundação, seus dois primeiros anos foram focados no desenvolvimento das plataformas de business to consumer (B2C) e business to business (B2B). Em novembro de 2006, a empresa deu início à estratégia de precificaçăo de acordo com o mercado. Em 2008, realizou um grande investimento em midia online para atração de

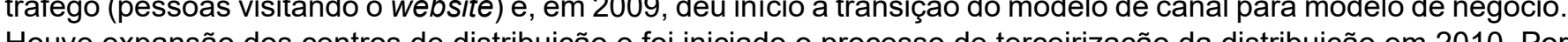
fim, em 2018, deu incio aos pimeios movimentos para se consolidar com aponta os principais movimentos da empresa Alfa.

Figura 1. Cronologia do e-commerce da empresa Alfa

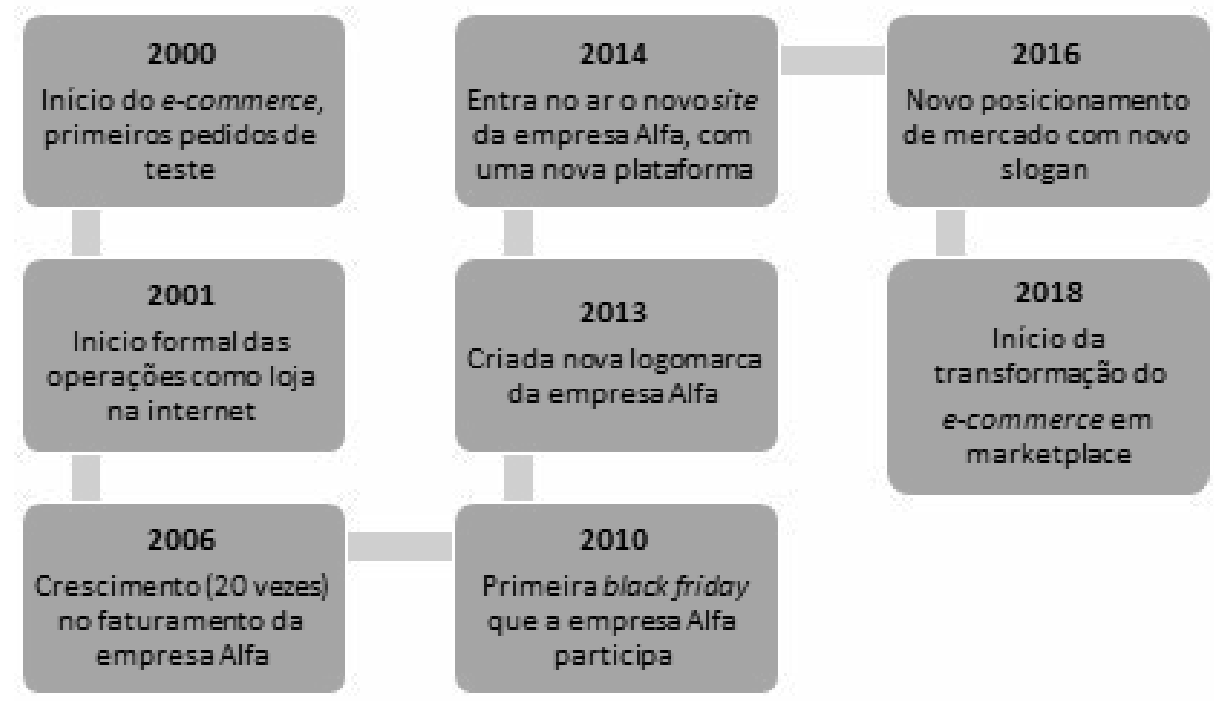

Fonte: Elaborado com base em Alfa (2018)

O e-commerce oferece mais de 15 mil produtos entre categorias como eletroeletrônicos, telefonia, informática, utilidades domésticas, alimentos, bebidas, material de construção, entre outros. Além da plataforma digital, os produtos também são oferecidos via telefone, chat e redes sociais. Seu grande diferencial consiste na sua própria origem - faz parte de um grupo atacadista com diversos centros de distribuição localizados no país, o que faz com que a Alfa consiga atender quase todo o território nacional. Esse grupo atacadista faturou R\$ 4,9 bilhões em 2018 (Abad, 2019).

Em entrevista, a relevância do tema justifica-se pela tendência de evolução dos marketplaces e também dos consumidores brasileiros, que buscam a comodidade, a redução dos riscos e a conveniência de buscar todos os produtos em um único lugar. Do ponto de vista dos grandes players do marketplace, a vantagem está em diversificar seu mix de produtos e aumentar o tráfego de visitantes e a frequência de compras no website.

Além do próprio e-commerce, a empresa Alfa vende seus produtos por meio de grandes marketplaces, entre os quais podem-se destacar: a) B2W: Iíder na América Latina, concentra operações de grandes empresas, como Americanas, Submarino, Shoptime e SouBarato. A plataforma, que vem sendo construída ao longo dos anos, oferece também serviços de tecnologia, logística, distribuição, atendimento ao cliente e pagamentos (B2W, 2017); b) Via Varejo: concentra operações da CasasBahia.com.br, Pontofrio.com, Extra.com.br e Barateiro.com. (Via Varejo, 2018); c) Amazon.com: operada pela Amazon Serviços de Varejo do Brasil Ltda., é uma afiliada da Amazon.com, que integra a lista de empresas Fortune 500 (Amazon, 2018); d) Wallmart: presente no Brasil desde 1995, possui lojas nos formatos hipermercado, supermercado, atacado, lojas de vizinhança e clube de compras (Wallmart, 2018).

Atuando por meio desses players, a empresa Alfa compra e armazena seu proprio estoque, para então vender, ou a venda ocorre por meio de cross docking, quando a empresa recebe o pedido e, então, compra o produto. A empresa não trabalha com o processo de drop ship, quando o produto sai da indústria direto para o consumidor. Dessa forma, toda a operação logística passa pela estrutura física da empresa Alfa.
Explorando um pouco mais as práticas realizadas pelas empresas dentro do marketplace, o entrevistado firma que a estratégia primária das corporações que oferecem essa estrutura é o aumento de tráfego, mas a grande vantagem está na rentabilização das operações a partir do aumento da eficiência aumento

Nesse contexto, as empresas maiores possuem um overhead de custos maiores, o que onera as vendas de produtos com baixa margem. Os sellers menores, muitas vezes, são mais eficientes em determinadas categorias. Assim, mesmo o grande player tendo maior poder de barganha na compra de determinados produtos, conseguindo um preço atrativo, a

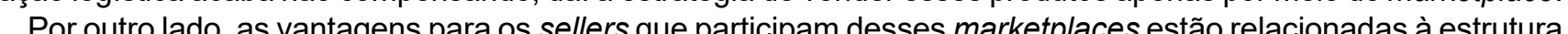
en de venda dos produco. Ao varã dén crédito por dos pros cred o, por ex pros

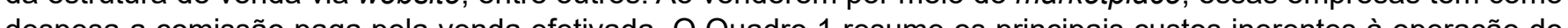
e-commerce versus marketplace.

Quadro 1. Comparação dos custos e-commerce vs marketplace

\begin{tabular}{|l|l|}
\hline \multicolumn{1}{|c|}{ Venda direta (e-commerce) } & \multicolumn{1}{c|}{ Venda por marketplace } \\
\hline Custos de marketing & \\
Custos operacionais (tarifas de cartão de crédito, \\
boleto bancário etc.) \\
Custos com análise antifraude \\
$\begin{array}{l}\text { Despesas com fraudes sofridas } \\
\text { Custos de manutenção do website }\end{array}$ & Comissão sobre venda realizada \\
\hline
\end{tabular}

Fonte: Elaboração a partir da entrevista

Em relação aos produtos vendidos nessa estrutura, todas os processos operacionais e logísticos ficam por conta dos sellers: a gestão dos estoques, os custos de entrega, as devoluções, as garantias, entre outros. Entretanto, para participar do marketplace, esses sellers precisam se adaptar às políticas e regras determinadas pelos primeiros, que são expressos por meio de contratos.

O entrevistado destaca que os contratos comerciais entre os sellers e os marketplaces estabelecem as regras do mercado. Esse contrato geralmente é um texto padrão, no qual estão descritas as condições de pagamento de comissão, as regras de participação, de respostas a possíveis reclamações de clientes, os prazos de entregas, os estoques, entre outros. Em geral, as cláusulas desses contratos são determinadas pelos marketplaces e, na maioria das vezes, não são passíveis de flexibilização.

As tabelas de comissão são divulgadas para os sellers, que precisam seguir as regras. Do contrário, podem ser descadastrados da plataforma. Os sellers só conseguem poder de barganha nessas negociações caso tragam algum diferencial a favor dos marketplaces, tais como exclusividade em determinada categoria ou grandes volumes de vendas. AAlfa se enquadra como um fornecedor importante, e está sempre entre os cinco melhores dos marketplaces em que atua (Alfa, 2018).

É esse poder de barganha que também determina a forma que o marketplace oferece a estrutura de atendimento e apoio aos sellers. No caso da Alfa, há um gestor de conta exclusivamente indicado para atendimento ao contrato. Para sellers menos importantes, não há um canal de atendimento dedicado, geralmente enviam a solicitação via e-mail e aguardam até que o marketplace responda às solicitações. Do ponto de vista do marketplace, esse atendimento traz custos, de modo que, para sellers com baixa rentabilidade, não compensa manter essa estrutura.

Um fator importante a destacar é a integração entre os participantes desse mercado. A maioria dos marketplaces não aceita receber integração direta dos sellers, pois essa comunicação precisa ser desenvolvida e homologada para que funcione de forma efetiva. Assim, os sellers precisam contratar um intermediário que faça o papel de hub logístico. São empresas que oferecem um software que possibilita a comunicação entre o seller e o marketplace, por onde transacionam dados sobre estoque disponivel, preços, tabelas de frete, entre outros. Os marketplaces possuem uma lista com as empresas homologadas e os sellers escolhem uma delas para fazer essa integração.

No caso da Alfa, existe uma equipe interna responsável por garantir que as informações sejam enviadas de forma correta para o integrador. Essa equipe tambem e responsável por monitorar o pedido do início ao fim, e, a partir da previsão de entrega, tratar possíveis atrasos antes mesmo que o cliente final perceba. Todo o tracking do pedido é enviado por meio do integrador, para que o cliente acompanhe pelo marketplace.

Sobre a qualidade do serviço prestado, os marketplaces fazem acompanhamento dos contratos firmados com os sellers, para garantir que eles cumpram os acordos, evitando riscos à imagem de sua marca. Caso os indicadores não estejam dentro do acordado, os sellers podem ser descadastrados da plataforma. Os custos decorrentes das

Revista Ciências Adiministrativas, 26(1): 9382, 2020 
operações por falhas dos sellers já são descontados do pagamento da comissão. Os markeplaces também fazem acompanhamento de alguns indicadores, tais como net promoter score (NPS), mas geralmente não são divulgados ao mercado, pois fazem parte de sua estratégia de atuação.

De um modo geral, o entrevistado afirma que as vantagens das práticas de colaboração entre os fornecedores para os sellers vai depender muito dos diferenciais estratégicos que eles possuem, como categorias exclusivas, grande volume de venda ou eficiência logística. Do contrário, eles terão pouco poder de barganha e terão que aceitar içôes impostas pelos marketplaces, que, nesse caso, são bastante inflexiveis.

Aempindo estava iniciando, ainda 2018, o desenvolvimento de seu próprio marketplace. O principal objetivo dessa estratégia è a complementaça de forma competitiva. O website já está passando por ado buãos para que essa parceria seja possivel. A produlos de forna compeniva. O Website já está passando por adequaços para que essa parceria seja possivel. A empresa Na seção seguinte será realizada a discussão dos resultados desta pesquisa e de sua contribuição teórica.

\section{Discussão dos Resultados}

O estudo de caso permitiu observar aspectos importantes sobre a atuação em um modelo de marketplace. O primeiro aspecto relaciona-se com a estratégia de cadeia de suprimentos. Uma vez que há a participação efetiva do consumidor final nas características da compra, a customização ocorre a partir do pedido concluído (realizado e pago). Com isso, existe a postergação máxima, seguindo os requisitos especificados pelo cliente, sendo utilizada a padronizaç̃o customizada apenas a partir da montagem do pedido e da distribuicão. Além disso, os marketplaces buscam melhorar sua eficiência por meio da parceira com os sellers, reduzindo custos operacionais e eliminando funções que não agregam valor.

O segundo aspecto é sobre as práticas colaborativas. Os marketplaces estabelecem parcerias com fornecedores, seja para aumentar o tráfego de visitantes, seja para melhorar sua eficiência logística, seja para diversificar seu mix de produtos, como é o caso da Alfa. Do ponto de vista dos sellers, essas parcerias permitem a redução de custos organizacional aumento das vendas e giro de mercadorias. Os sellers e os players do marketplace possuem estrutura definem as regras e os papéis de cada participante. $\mathrm{O}$ terceiro aspecto é a dependência do uso de tecnologia para permitir o funcionamento do modelo. Isso pode ser observado pela necessidade de intermediação entre os sellers e os marketplaces, realizado pelas empresas integradoras. Essas empresas fornecem softwares indispensáveis para o funcionamento eficienégi. que permite a troca e a gestão das informações, sem o qual não seria possivel essa estratégia.

O quarto e último aspecto diz respeito ao serviço ao cliente. Em relação aos elementos da pré-transação, o estudo de caso mostrou que a parceria entre os sellers e os marketplaces é firmada por meio de contratos, nos quais Há um acompanhamento do cumprimento dos critérios acordados pelos marketplaces, que podem descadastrar os fornecedores em caso de não cumprimento. Os fornecedores mais importantes possuem mais condições de negociar as cláusulas desses contratos, bem como os valores de comissão praticados, e ainda possuem um canal de atendimento específico para suas necessidades.

Em relação aos elementos da transação, todas as informações de estoques, preços, condições de venda e acompanhamento do pedido até a entrega são transacionadas via integração sistêmica. Existem empresas homologadas, responsáveis por essa integração, para garantir a eficiência do processo. As condições da operação também estão firmadas em contrato, e há o acompanhamento dos níveis de serviço pelo marketplace. Embora toda a operação logística esteja na gestão do fornecedor, os custos decorrentes do não cumprimento das regras definidas em contrato são descontados da comissão paga pelo marketplace. Entretanto todo o processo de efetivação da venda, divulgação dos produtos, análise antifraude, manutenção da estrutura e apoio ao cliente ficam sob a gestão do marketplace.

Por fim, em relação aos elementos da pós-transação - a política de devoluções, a logística reversa, a garantia de entrega dos produtos, são determinados pelo marketplace (também via contratos) e devem cumpridos pelos sellers. Os custos do não cumprimento também são descontados das comissões pagas pelas vendas, e a reincidência de falha pode incorrer em descadastramento do fornecedor.

Todos os aspectos elencados aqui respondem à pergunta proposta neste estudo, ou seja, explicam como se dá o processo de serviço ao cliente a partir da estratégia de marketplace.

\section{Considerações Finais}

Este estudo teve como objetivo avaliar a importância do serviço ao cliente no modelo de marketplace, no contexto das estrategias da cadeia de suprimentos. Nesse sentido, desenvolveu-se uma revisão bibliográfica sobre as estratégias voltadas para o atendimento ao cliente, as práticas de colaboração entre fornecedores e também a forma como o serviço logístico é avaliado dentro do intervalo de tempo - antes, durante e após as operações transacionais. O estudo de caso contribuiu para a compreensão de aspectos sobre a atuação das empresas dentro de um modelo de marketplace, tema ainda pouco explorado na literatura existente. Nesse sentido, o manuscrito explorou os seguintes aspectos. primeiramente, a avaliação dos fluxos de produção empurrado e puxado na cadela de suprimentos, viabilzando-se mecanismos de postergação, a necessidade de avaliação da colaboraçáo entre os agentes da cadeia que vizizar a ina que apolam a integração dos agentes do marketplace e, por fim, o aspecto de avallação das operaçoes comerciais

Diante dos apontamentos, comprovou-se que a estratégia de marketplace está alinhada à definição de que a qualidadeno destacado nas dimensões do design da interface entre f́c

Costudo de caso único, que contemplou exclusiva Destaca-se também, a contribuicão social visto as possibilidas para pequenas organizaçes act

cados com barreiras, seja por questões financeiras, logísticas ou de marketing.

\section{Referências}

Abad. (2019). ABAD - Associação Brasileira de Atacadistas Distribuidores de Produtos Industrializados. Link

Aguiar. F. H. O. D. \& Sampaio M. (2014). Identificação dos fatores que afetam a ruptura de estoque utilizando análise de agrupamentos. Production, 24(1), 57-70.

Alfa. (2018). Quem Somos. Link

Amazon. (2018). Get to know us - About Amazon. Link

B2W. (2017). Comércio Eletrônico no Brasil IInstitucional / B2W Compania Digital. Link

Bardin, L. (1977). L'Analyse de contenu. Paris: Presses Universitaires de France.

Ballou, R. (1993). Logística empresarial: Transportes, administração de materiais e distribuição física. São Paulo: Atlas.

Bezes, C. (2016). Comparing online and in-store risks in multichannel shopping. International Journal of Retail \& Distribution Management, 44(3), 284-300.

Bhatnagar, A., \& Ghose, S. (2004). A latent class segmentation analysis of E-shoppers. Journal of Business Research, 57, 758-767.

Boni, V., \& Quaresma, S. J. (2005). Aprendendo a entrevistar: Como fazer entrevistas em Ciências Sociais. Revista Eletrônica de Pós Graduandos em Sociologia Política da UFSC, 2(1),68-80.

Bowersox, D., \& Closs, D. (2001). Logística empresarial: O processo de integração da cadeia de suprimento. São Paulo: Atlas.

Branski, R. M., \& Laurindo, F. J. (2013). Tecnologia da informação e integração das redes logísticas. Gestão e Produção, 20(2), 255-270.

Carvalho, G., \& Campos, R. (2013). Análise dos benefícios do EDI para a competitividade logística das organizações. Gestão Industrial, 8(4), 105-120.

Cheah, I., Phau, I., \& Liang, J. (2015). Factors influencing consumers' attitudes and purchase intentions of e-deals. Marketing intelligence \& planning, 33(5), 763-783.

Collier, J., \& Bienstock, C. (2006). Measuring service quality in E-retailing. Journal of Service Research, 8(3), 260-275. 
Collis, D., \& Montgomery, C. (Jul/Aug 1995). Competing on resources: Strategy in the 1990s. Harvard Business Review, (4), 118-128.

Daugherty, P. J., Bolumole, Y., \& Grawe, S. J. (2019). The new age of customer impatience: An agenda for reawakening logistics customer service research. International Journal of Physical Distribution \& Logistics Management, 49(1), 4-32.

Davis, B. R., \& Mentzer, J. T. (2006). Logistics service driven loyalty: An exploratory study. Journal of Business Logistics, 27(2), 53-73.

Donmoyer, L. (2009). Principal perceptions of their preparedness and the preparedness of their staff to deal with students of proverty in the classroom. Dissertation in EdD Education Leadership, Management and Policy, Seton Hall University, South Orange, Nova Jersey. Link

Durski, G. (2003). Avaliação do desempenho em cadeias de suprimentos. Revista da FAE, Curitiba, 6(1), 27-38.

Ebit. (2018). Webshoppers. Link

Figueira, A., \& Buri, M. R. (2017). Os benefícios da utilização do sistema warehouse management system na cadeia de logística reversa no brasil. Exacta, 15(2), 245-257.

Fisher, M. L. (1997). What is the right suply chain for your product. Harvard Business Review, 75, 105-116.

Gil, A. C. (2002). Como elaborar projetos de pesquisa (4a ed). São Paulo: Atlas.

Guissoni, L. A., \& Farinha, R. L. (2019). E-commerce com resultado. GV-executivo, 18(1), 40-42.

Gomes, L. G., \& Kliemann Neto, F. J. (2015). Métodos colaborativos na gestão de cadeias de suprimentos: Desafios de implementação. RAE - Revista de Administração de Empresas, 55(5). DOI: 10.1590/S0034-759020150508

Handfield, R., \& Nichols, E. (1999). Introduction to supply chain management. New Jersey: Prentice Hall.

Hayes, R. (2006). Operations, strategy and technology: Pursuing the competitive edge. Strategic Direction, 22(7).

Hilletofth, P. (2009). How to develop a differentiated supply chain strategy. Industrial Management \& Data Systems, 109(1), 16-33

Hong, I. B., \& Cho, H. (2011). The impact of consumer trust on attitudinal loyalty and purchase intetions in B2C e-marketplaces: Intermediary trust vs. seller trust. International journal of information management, 31(5), 469-479.

Hsu, M. K., Huang, Y., \& Swanson, S. (2010). Grocery store image, travel distance, satisfaction and behavioural intentions. International Journal of Retail and Distribution Managemen, 38(2), 115-132.

Kubota, L. C., \& Milani, D. N. (2011). Os efeitos do e-commerce na produtividade das firmas comerciais no Brasil. Link

Lambert, D. M. (2006). Suply chain management: Process, partnerships, performance (2a ed). Sarasota: Suply Chain Management Institute.

Leuschner, R., Charvet, F., \& Rogers, D. S. (2013). A meta-analysis of logistics customer service. Journal of supply chain management, 49(1), 47-63.

Lichtenstein, D. R., Ridgway, N. M., \& Netemeyer, R. G. (1993). Price Perceptions and consumer shopping behavior: A field study. Journal of marketing research, 30(2), 234-245.

Marques, É. V. (2010). Aspectos da logística no comércio eletrônico business-to-consumer: um estudo de caso no varejo brasileiro. Revista Ciências Administrativas ou Journal of Administrative Sciences, 8(2).

Mazzali, L., \& Padilha, R. (2006). O comércio eletrônico e a competência logística: Estudos de casos em pequenas 10 empresas varejistas. Gestão \& Regionalidade, 22(63), 69-82.

Mentzer, J. T., Flint, D. J., \& Hult, G. T. (2001). Logistics service quality as a segment-customized process. Journal of Marketing, 65(4), 82-104.

O'Cass, A., \& Carlson, J. (2012). An e-retailing assessment of perceived website-service innovativeness: Implications for website quality evaluations, trust, loyalty and word of mouth. Australasian Marketing Journal, 20(1), 28-36.

Oliveira, D. (2001). Planejamento estratégico, conceitos, metodologias e práticas. São Paulo: Atlas.

Pigatto, G., \& Alcantara, R. (2007). Relacionamento colaborativo no canal de distribuição: Uma matriz para análise. Gestão e Produção, 14(1), 155-167.

Rafele, C. (2004). Logistic service measurement: A reference framework. Journal of Manufacturing Technology Management, 15(3), 280-290

Reinartz, W., Dallaert, B., Krafft, M., Kumar, V., \& Varandarajan, R. (2011). Retailing innovations in a globalizing retail market. Journal of Retailing, 87, 53-66.

Rosenzweig, E., \& Easton, G. S. (2010). Tradeoffs in Manufacturing? A meta-analysis and critique of the literature. Production and Operations Management, 19(2), 127-141.

Saghiri, S., Wilding, R., Mena, C., \& Bourlakis, M. (2017). Toward a three-dimensional framework for omni-channel. Journal of Business Research, 77, 53-67.

Shankar, V., Smith, A. K., \& Rangaswamy, A. (2003). Customer satisfaction and loyalty in online and offline environments. International Journal of Research in Marketing, 20(2), 153-175.

Sociedade Brasileira de Varejo e Consumo [SBVC]. (2018). 50 Maiores Empresas E-commerce Brasileiro. Link

Silva, E., \& Menezes, E. (2005). Metodologia da pesquisa e elaboração de dissertação (4a ed. rev. atual. ed.). Florianópolis: UFSC

Simão, L., \& Gonçalves, M. (2015). Sistemática para seleção de um portfólio de estratégias de postergação para cadeias suprimentos. GEPROS: Gestão da Produção, Operações e Sistemas, 10(1), 35-47.

Stank, T. P., Goldsby, T. J., \& Vickery, S. K. (1999). Effect of service supplier performance on satisfaction and loyalty of store managers in the fast food industry. Journal of Operations Management, 17(4), 429-447.

Stank, T. P., Pellathy, D. A., In, J., Mollenkopf, D. A., \& Bell, J. E. (2017). New frontiers in logistics research: Theorizing at the middle range. Journal of Business Logistics, 38(1), 6-17.

Stuart, I., McCutcheon, D., Handfield, R., McLachin, R., \& Samson, D. (2002). Effective case research in operations management: A process peerspective. Journal of Operations Management, 20(5), 419-433. DOI: 10.1016/ S0272-6963(02)00022-0

Turban, E. E., Lee, J. K., King, D., Liang, T. P., \& Turban, D. (2009). Eletronic commerce 2010: A managerial perspective. New Jersey: Prentice Hall.

Turban, E., \& King, D. (2004). Comércio eletrônico: Estratégia e gestão. São Paulo: PrenticeHall.

Van Hoek, R. I. (2001). The rediscovery of postponement: A literature review and directions for research. Journal of operations management, 19(2), 161-184

Via Varejo. (2018). Market Place Via Varejo - Soluções e Serviços. Link

Vieira, V. A., Matos, C. A., \& Slongo, L. A. (2009). Avaliação das relações entre qualidade de serviço do site, satisfação, valor percebido, lealdade e boca a boca por meio de um modelo teórico. Revista de Administração USP, 44(2), 131-146.

Revista Ciências Adiministrativas, 26(1): 9382, 2020 
Wallmart. (2018). Wallmart no Brasil. Link

Whipple, J. M, \& Russel, D. (2007). Building supply chain collaboration: A typology of collaborative approaches. The International Journal of Logistics Management, 18(2), 174-196.

Yang, B., \& Burns, N. (2003). Implications of postponement for the supply chain. International journal of production research, 41(9), 2075-2090.

Yu, Y., Wang, X., Zhong R. Y., \& Huang, G. Q. (2016). E-commerce logistics in supply chain management: Practice perspective. Procedia Cirp, 52, 179-185.

Yin, R. (2015). Estudo de caso: Planejamento e métodos. Porto Alegre: Bookman.

Zilber, S. N., \& Rosa, M. S. de. (2014). Adoção de e-business por indústrias: Mudanças na logística e na estrutura organizacional. Revista Gestão Organizacional, 6(2), 56-70.

\section{Contato:}

Renata de Souza Franco

E-mail: renatasf@live.com

Leonardo Caixeta de Castro Maia

E-mail: leonardocaixeta.maia@gmail.com

Rafael Gontijo de Souza Filho

E-mail: rafaelfgontijo@hotmail.com 